\title{
L'augmentation des teneurs en delta-9 tétrahydrocannabinol dans les produits à base de cannabis en France : mythe ou réalité ?
}

\author{
Is the increase of delta-9 \\ tetrahydrocannabinol in street drugs \\ documented by analytical findings?
}

\begin{abstract}
Patrick MURA $^{(1)}$, Martine PERRIN ${ }^{(2)}$, Martine CHABRILLAT ${ }^{(3)}$, Huguette CHAUDRON ${ }^{(4)}$, Véronique DUMESTRE-TOULET ${ }^{(5)}$, Stéphanie BARC ${ }^{(1)}$, Gilbert PÉPIN ${ }^{(6)}$
\end{abstract}

(1) Laboratoire de Biochimie et Toxicologie, Centre Hospitalier Universitaire - BP 577 - 86021 POITIERS

(2) Institut de Recherche Criminelle de la Gendarmerie Nationale - ROSNY-SOUS-BOIS

(3) Laboratoire Interrégional des Douanes - PARIS

(4) Laboratoire de Police Scientifique - LYON (5) Laboratoire BIOffice - ARTIGUES-PRÈS-BORDEAUX (6) Laboratoire Toxlab - PARIS

(Reçu le 17 avril 2001 ; accepté le 25 mai 2001)

\section{RÉSUMÉ}

L'augmentation des teneurs en THC dans les produits à base de cannabis vendus en France est, depuis quelques années, fréquemment évoquée dans la littérature. Cependant, à notre connaissance, cette affirmation ne repose sur aucune base bibliographique. L'objet de la présente étude a été de vérifier la valeur de cette information.

Nous avons rassemblé 5152 résultats d'analyses obtenus de 1993 à 2000 sur des produits de saisies effectuées par les services des douanes, de la police nationale et de la gendarmerie nationale. Les renseignements fournis indiquaient l'année de la saisie, le type de produit et la teneur observée en delta-9 tétrahydrocannabinol (THC). Les analyses étaient réalisées par chromatographie en phase gazeuse couplée à la spectrométrie de masse.

Les résultats indiquent que, quelle que soit l'année étudiée, une très grande variabilité des concentrations était observée pour "l'herbe" mais aussi pour la "résine". Des concentrations en THC inférieures à $2 \%$ étaient relativement fré-

\section{SUMMARY}

The increase in the delta-9 tetrahydrocannabinol (THC) content of cannabis sold in France has been frequently claimed in the literature for several years. However, according our knowledge, this assessment has never been documented. The purpose of the present study was to evaluate the value of this assertion. We have compiled 5152 results obtained from 1993 to 2000 on seized cannabis products by the laboratories of French customs, national police force, and from some private forensic toxicology laboratories. Each result indicated the year of the seizure, the strain of cannabis and the THC content. The analyses were performed by gas chromatography - mass spectrometry.

Irrespective the year, very important variations were observed in THC contents for herb as well as for resin. THC levels lower than $2 \%$ were relatively frequent (18\% of samples). Up to 1995, $75 \%$ of herbal samples contained less than $5.5 \%$ of THC, and the maximum value found in a French seizure was $8.7 \%$. During the same period, $47 \%$ of resin samples contained between 5 and $10 \%$ of THC. Since 1996, 
quentes (18\% des échantillons). Jusqu'en 1995, $75 \%$ des échantillons d'herbe contenaient moins de 5,5\% de THC, et la teneur la plus élevée observée dans une saisie a été de $8,7 \%$. Pendant la même période, $47 \%$ des échantillons de résine contenaient entre 5 et $10 \%$ de THC. Depuis 1996, bien que d'une façon générale les teneurs observées dans la majorité des échantillons n'aient pas beaucoup évolué $(54 \%$ des échantillons d'herbe et $71 \%$ des échantillons de résine contenaient respectivement moins de $5 \%$ et moins de $10 \%$ de THC), sont apparus de façon exceptionnelle des échantillons à base de cannabis très fortement concentrés en THC. Au cours de l'année 2000, $3 \%$ des échantillons d'herbe et $18 \%$ des échantillons de résine analysés contenaient plus de $15 \%$ de THC. Ces échantillons très concentrés correspondaient le plus souvent aux nouveaux produits comme la "skunk", la "super skunk" ou le "pollen".

En conclusion, il y a eu depuis 1996 une augmentation des teneurs en THC avec notamment l'apparition de nouveaux produits très fortement titrés, mais cette évolution n'a concerné qu'une minorité des échantillons disponibles sur le marché clandestin français.

\section{MOTS-CLÉS}

Cannabis, delta-9 tétrahydrocannabinol, THC.

\section{Introduction}

Parmi la soixantaine de cannabinoïdes présents dans Cannabis sativa indica, le $\Delta^{9}$-transtétrahydrocannabinol (THC) constitue le principal produit psychoactif chez l'homme.

Barnett et coll (1) ont étudié, sur simulateurs de conduite, les effets (erreurs de conduite) obtenus par la consommation de "joints" dosés à 100, 200 ou $250 \mu \mathrm{g} / \mathrm{kg}$ de poids du sujet et comparé ces résultats aux concentrations sanguines en THC. Ils ont indiqué l'existence d'une corrélation linéaire significative pendant une durée de 2 à $7 \mathrm{~h}$ (selon les effets, le plus persistant étant le suivi de trajectoire). Sur la base des travaux de Cocchetto et coll (2), Harder et coll (3) ont comparé l'évolution dans le temps des concentrations sanguines de THC et les effets psychiques ressentis par les sujets, après consommation de "joints" contenant $9 \mathrm{mg}$ de THC (joint "standard" tel qu'il est défini aux Etats-Unis par le National Institute on Drug Abuse), $3 \mathrm{mg}$ ou $1 \mathrm{mg}$. Ils ont ainsi indiqué que les effets psychiques obtenus après consommation isolée d'un joint contenant $9 \mathrm{mg}$ de THC persistent pendant une durée d'environ $2 \mathrm{~h}$, tandis que la concentration en THC dans le sang est rapidement très faible et de l'ordre $\mathrm{du} \mathrm{ng} / \mathrm{ml}$ au bout de $2 \mathrm{~h}$. Les mêmes auteurs ont par ailleurs montré que l'amplitude des effets était dépendante de la dose et de la concentration sanguine maximale observée.

De ces travaux, il résulte donc que les effets pharmacologiques du THC (modifications des perceptions senso- although THC levels of most of the samples have not signifcantly increased $(54 \%$ of herbal samples and $71 \%$ of resin samples contained respectively less than 5 and $10 \%$ of THC), more potent cannabis samples exceptionally appeared. During the 2000 year, $3 \%$ of herbal products and $18 \%$ of resin samples contained more than $15 \%$ of THC. These seizures consisted generally in new strains such as "skunk", "super skunk" or "pollen".

As a conclusion, since 1996 there has been an increase in the THC content of cannabis with the exceptional occurrence of very potent strains, but this evolution concerns only a minority of products that are available in France.

\section{KEY-WORDS \\ Cannabis, delta-9 tetrahydrocannabinol, THC.}

rielles, troubles thymiques et dissociatifs, levée des inhibitions, altérations de la mémoire à court terme, etc.) chez un sujet donné seront d'autant plus importants que le produit utilisé contient un pourcentage élevé en THC.

Grâce à des techniques horticoles très avancées (cultures sous serres, hydroponiques, avec des conditions de luminosité et de température optimales), les néerlandais réussissent désormais à obtenir des variétés à très forte teneur en THC (4).

Ces dernières années, l'usage de cannabis a été directement mis en cause en France dans de nombreux cas médico-légaux : accidents de la circulation routière (56), défenestration après consommation d'un seul joint, etc. Des effets délétères très prononcés (états délirants aigus, hallucinations, crises de panique) sont de plus en plus souvent rapportés dans la littérature (7).

De nombreux auteurs expliquent ces observations par une augmentation très importante au cours de la dernière décennie des teneurs en $\mathrm{THC}$ dans les produits à base de cannabis vendues en France. Cette affirmation est devenue une constante dans la littérature (4-7-9). Cependant, à notre connaissance, aucune étude n'a été à ce jour réalisée en France sur ce thème.

\section{Matériel et méthodes}

Nous avons rassemblé 5152 résultats d'analyses obtenus depuis 1993 sur des produits de saisies effectuées en France par le laboratoire Interrégional des douanes 
(Paris), les 5 laboratoires de police scientifique (Lyon, Marseille, Lille, Toulouse, Paris), le laboratoire de l'Institut de Recherches Criminelles de la Gendarmerie Nationale (Paris), le laboratoire Toxlab (Paris) et le laboratoire BIOffice (Artigues-Près-Bordeaux). Les renseignements fournis indiquaient l'année de la saisie, le type de produit (herbe, résine, etc.) et la teneur observée en THC. Les analyses ont été réalisées par chromatographie en phase gazeuse couplée à la spectrométrie de masse et les résultats étaient exprimés en pourcentages par rapport au poids de matière sèche.

\section{Résultats}

Les évolutions des teneurs en THC observées dans les produits de saisies en France depuis 1993 pour "l'herbe" et pour la "résine" sont respectivement présentées dans les Figures 1 et 2. L'évolution des concentrations maximales est indiquée dans le tableau I.

Quelque soit l'année étudiée, une très grande variabilité des concentrations en THC était observée, aussi bien pour "l'herbe" que pour la "résine", et des concentrations inférieures à $2 \%$ étaient relativement fréquentes (18\% des échantillons).

Jusqu'en 1995, les $2 / 3$ des échantillons d'herbe contenaient moins de $5 \%$ de principe actif et la teneur la plus élevée observée dans une saisie a été de $8,7 \%$. Pendant la même période, près de la moitié $(47 \%)$ des échantillons de résine contenaient entre 5 et $10 \%$ de THC et la plus forte teneur a été de $10,6 \%$.

De 1996 à 1998, apparurent sur le marché clandestin des échantillons d'herbe contenant entre 10 et $15 \%$ de THC (concernant environ 1 saisie sur dix) ainsi que de la résine titrant à plus de $15 \%$ (concernant $8 \%$ des saisies).

Au cours des années 1999 et 2000 , d'une façon générale les teneurs observées dans la majorité des échantillons n'ont pas beaucoup évolué : $54 \%$ des saisies d'herbe contenaient moins de $5 \%$ de THC et $71 \%$ des échantillons de résine comportaient moins de $10 \%$ de principe actif. En revanche, sont apparus des échantillons très fortement titrés, avec des teneurs en THC pouvant atteindre $20 \%$ pour l'herbe et $30 \%$ pour la résine. Les concentrations en principe actif les plus élevées provenaient des "nouveaux produits" que sont la "skunk" et la "super skunk" (variétés de fleurs de cannabis originaires initialement des U.S.A. et désormais des Pays-Bas), le "pollen" (étamines de plants mâles). Il est à noter que ces échantillons étaient rencontrés en France de façon exceptionnelle puisqu'ils représentaient environ $1 \%$ des saisies effectuées en 1999 et 2000.

\section{Échantillons} (\%)

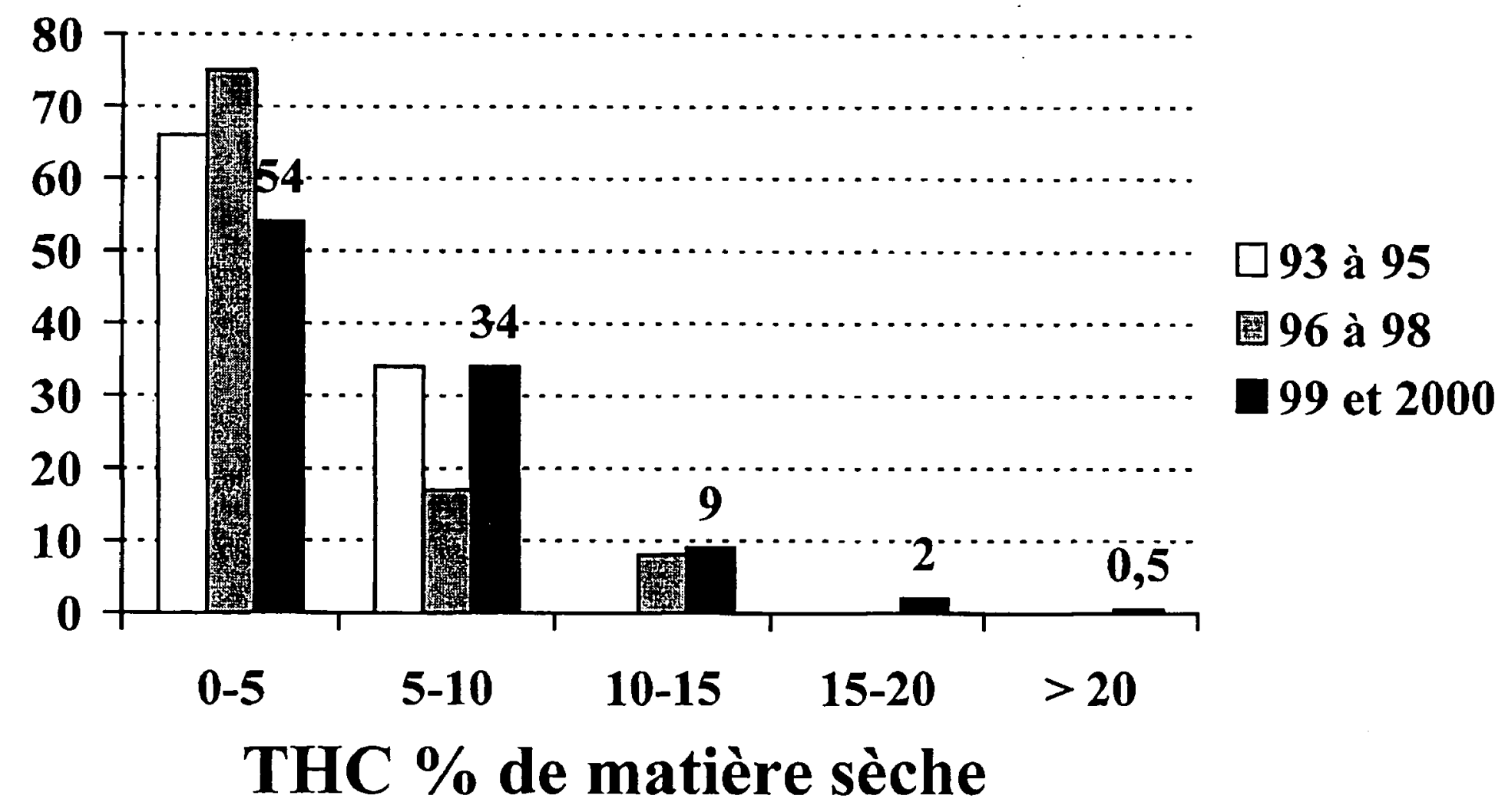

Figure 1 : Évolution des teneurs en THC observées en France pour l' "herbe” de 1993 à 2000. 


\section{Échantillons}

(\%)

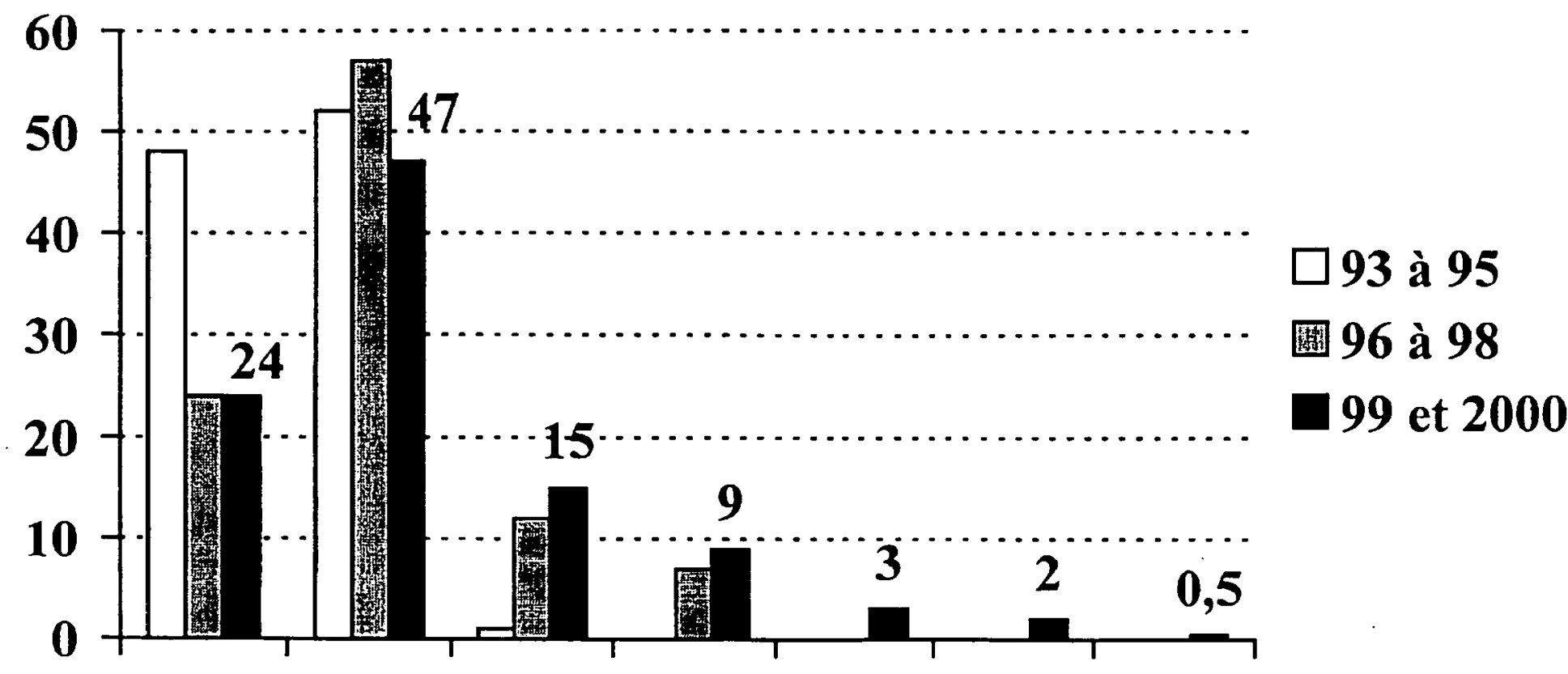

\section{$\begin{array}{llllllll}0-5 & 5-10 & 10-15 & 15-20 & 20-25 & 25-30 & >30\end{array}$ THC \% de matière sèche}

Figure 2 : Évolution des teneurs en THC observées en France pour la "résine" de 1993 à 2000.

Tableau I : Teneurs maximales en THC observées en France, exprimées en pourcentage de poids de matière sèche.

\begin{tabular}{|c|c|c|c|c|c|c|c|c|}
\hline & $\mathbf{1 9 9 3}$ & $\mathbf{1 9 9 4}$ & $\mathbf{1 9 9 5}$ & $\mathbf{1 9 9 6}$ & $\mathbf{1 9 9 7}$ & $\mathbf{1 9 9 8}$ & $\mathbf{1 9 9 9}$ & $\mathbf{2 0 0 0}$ \\
\hline Herbe & 5,5 & 5,9 & 8,7 & 14,4 & 19 & 22,9 & 23,6 & 21,7 \\
\hline Résine & 10,6 & 9,8 & 10,2 & 15,8 & 22,7 & 26,7 & 41,3 & 31,7 \\
\hline
\end{tabular}

\section{Discussion et conclusion}

Il a été établi que, dans les préparations à base de cannabis, le contenu en delta-9 tétrahydrocannabinol diminuait avec le temps, le THC s'oxydant en cannabinol (10). Il a cependant été démontré récemment que cette dégradation n'était pas quantitativement très importante, de l'ordre de $1 \%$ (11); de tels phénomènes de dégradation éventuelle ne devraient donc pas avoir influé de façon significative sur l'interprétation des résultats.

Une étude australienne récente (12), portant sur l'analyse de 31000 produits de saisies effectuées dans ce pays entre 1980 et 1996, a révélé que les moyennes des teneurs ont augmenté mais de façon très modérée, passant de $1,2 \%$ en 1980 à 4,2\% en 1996. Ces auteurs en concluaient qu'il y avait un décalage entre les affirmations de certains "commentateurs" et ces résultats. Au plan méthodologique, nous n'avons pas choisi pour notre étude d'analyser les variations dans le temps en utilisant comme paramètre les teneurs moyennes annuelles. Ce paramètre nous a semblé en effet être de nature à fausser l'interprétation, certaines saisies ayant été effectuées sur des cultures sauvages locales dont un certain nombre concernaient des plants immatures, qui n'auraient donc pas été commercialisés à ce stade. Quoiqu'il en soit, nos résultats concernant la France corroborent les conclusions de l'étude australienne, montrant que les teneurs en THC dans les produits de saisie effectuées en France ont très peu évolué jusqu'en 1996. Depuis cette date en revanche, des teneurs supérieures à 10 voire $15 \%$ sont apparues. Cela est particulièrement notable pour la résine. En effet, si jusqu'en $19952 \%$ des échantillons de résine contenaient plus de $10 \%$ de THC, ils étaient $20 \%$ de 1996 à 1998 et environ $30 \%$ en 1999 et 2000.

La très grande variabilité observée, selon les saisies, des concentrations en principe actif confirme l'extrême difficulté à évaluer la consommation réelle d'un individu à partir du nombre de "joints" fumés (13). Il appa- 
raît encore plus illusoire de vouloir en déduire les effets psychoactifs sur le consommateur, la seule possibilité pour ce faire demeurant l'analyse sanguine par méthode chromatographique $(6,14)$.

La consommation des nouveaux produits à très forte teneur en THC (supérieure à $20 \%$ ) a souvent été rendue responsable de l'observation de plus en plus fréquente de certaines symptomatologies telles que des hallucinations visuelles ou auditives, attaques de paniques ou autres états psychotiques aigus (4). A la lumière de nos résultats, de tels échantillons ne sont qu'exceptionnellement rencontrés. Il apparaît plus probable que ces effets intenses soient liés à des contextes de consommation différents de ceux qu'ils étaient généralement il y a une dizaine d'années : âge des consommateurs de plus en plus en plus jeune, consommation quantitativement importante à la recherche de sensations fortes, etc.

En conclusion, dire que "le cannabis d'aujourd'hui n'a rien de comparable à celui des années 1970" est à la fois un mythe et une réalité. Un mythe car au cours de ces deux dernières années, $71 \%$ des échantillons de résine et $88 \%$ des échantillons d'herbe contenaient moins de $10 \%$ de THC. Une réalité car depuis 1996, sont apparus de façon exceptionnelle sur le marché clandestin français des échantillons fortement à très fortement titrés en principe actif.

Le danger que constitue l'usage de cannabis pour soimême (désocialisation, pertes de mémoire à court terme, etc.) et pour autrui (accidents de la circulation routière, comportement à risque en milieu de travail, etc.) ne semble donc pas être une conséquence de l'évolution des teneurs en principe actif. Ce danger pourtant réel, comme l'attestent des observations de plus en plus fréquentes notamment en matière de sécurité routière (15), serait beaucoup plus à associer au fait que le cannabis est un produit caractérisé par une forte toxicité neuro-comportementale, dont les effets psychoactifs peuvent s'exprimer chez certains sujets (et tout particulièrement chez les jeunes) même avec des doses considérées comme faibles.

\section{Références}

1. Barnett G., Licko V., Thompson T. Behavioral pharmacokinetics of marijuana. Psychopharmacology (berl) $1985 ; 85: 51-56$.

2. Cochetto D.M., Owens S.M., Perez-Reyes M., Diguiseppi S., Miller L.L. Relationship between plasma delta-9 tetrahydrocannabinol concentration and pharmacologic effects in man. Psychopharmacology $1981 ; 75$ : 158-164.

3. Harder S., Rietbrock S. Concentration-effect relationship of delta-9-tetrahydrocannabiol and prediction of psychotropic effects after smoking marijuana. Int $\mathrm{J}$ Clin Pharmacol Ther $1997 ; 35: 155-159$.

4. Paris M., Tran N. The existence of "Nederwiet", a new fact in the history of cannabis. Ann Pharm Fr 1998 ; 56 : 264-267.

5. Mura P., Piriou A. Le cannabis. In : Mura P., ed. Alcool, médicaments, stupéfiants et conduite automobile. Paris : Elsevier, 1999 ; 143-169.

6. Kintz P., Cirimele V., Mairot F., Muhlmann M., Ludes B. Analyses toxicologiques pratiquées sur 198 conducteurs accidentés. Presse Med $2000 ; 29: 1275-1278$

7. Reynaud M., Parquet P..J., Lagrue G. In : Les pratiques addictives. Paris : Odile Jacob ed. 2000.

8. Aquatias S., Maillard I., Zormann M. Faut-il avoir peur du haschich ? Paris : Syros, 1999.
9. Rapport de l'Académie des Sciences. Aspects moléculaires, cellulaires et physiologiques des effets du cannabis. $\mathrm{n}^{\circ}$ 39. Paris : Lavoisier Technique et documentation, avril 1997.

10. El Kheir Y.M., Mohamed M.I., Hakim H.A. Fitoterapia $1986 ; 57: 235-237$.

11. Stefanidou M., Athanaselis S., Alevisopoulos G., Papoutsis J., Koutselinis A. Delta-9 tetrahydrocannabinol content in cannabis plants of greek origin. Chem. Pharm. Bull. $2000 ; 48: 743-745$.

12. Hall W., Swift W. The THC content of cannabis in Australia : evidence and implications. Aust N Z J Public Health $2000 ; 24: 503-508$.

13. Pélissier A.L., Léonetti G., Villani P., Cianfarani F., Botta A. Le cannabis : mise au point toxicocinétique et méthodologie du dépistage urinaire. Thérapie $1997 ; 52: 213-218$.

14. Kintz P., Cirimele V., Pépin G., Marquet P., Deveaux M., Mura P. Identification et dosage des cannabinoïdes dans le sang total. Toxicorama $1996 ; 8: 29-33$.

15. Mura P., Kintz P., Gaulier J.M., Vincent F. Goullé J.P., Moulsma M., Mairot F., Martin-Dupont S., Cadour A., Nouveau J., Tilhet-Coartet S., Pourrat O. Etude castémoins à inclusion prospective de l'usage de cannabis chez des conducteurs impliqués dans un accident corporel de la circulation routière. Résultats préliminaires obtenus chez 420 conducteurs et 381 sujets témoins. Annales de Toxicologie Analytique $2001 ; 13$ : 75-79. 